\title{
Absolutely convergent Fourier series. An improvement of the Beurling-Helson theorem
}

\author{
Vladimir Lebedev
}

\begin{abstract}
We consider the space $A(\mathbb{T})$ of all continuous functions $f$ on the circle $\mathbb{T}$ such that the sequence of Fourier coefficients $\widehat{f}=$ $\{\widehat{f}(k), k \in \mathbb{Z}\}$ belongs to $l^{1}(\mathbb{Z})$. The norm on $A(\mathbb{T})$ is defined by $\|f\|_{A(\mathbb{T})}=\|\widehat{f}\|_{l^{1}(\mathbb{Z})}$. According to the known Beurling-Helson theorem, if $\varphi: \mathbb{T} \rightarrow \mathbb{T}$ is a continuous mapping such that $\left\|e^{i n \varphi}\right\|_{A(\mathbb{T})}=$ $O(1), n \in \mathbb{Z}$, then $\varphi$ is linear. It was conjectured by Kahane that the same conclusion about $\varphi$ is true under the assumption that $\left\|e^{i n \varphi}\right\|_{A(\mathbb{T})}=$ $o(\log |n|)$. We show that if $\left\|e^{i n \varphi}\right\|_{A(\mathbb{T})}=o\left((\log \log |n| / \log \log \log |n|)^{1 / 12}\right)$ then $\varphi$ is linear.

References: 15 items.

Keywords: absolutely convergent Fourier series, Beurling-Helson theorem.
\end{abstract}

AMS 2010 Mathematics Subject Classification. 42A20

We consider the class $A(\mathbb{T})$ of all continuous functions $f$ on the circle $\mathbb{T}=\mathbb{R} / 2 \pi \mathbb{Z}$ whose Fourier series

$$
f(t) \sim \sum_{k \in \mathbb{Z}} \widehat{f}(k) e^{i k t}
$$

converge absolutely. Here $\mathbb{R}$ is the real line, $\mathbb{Z}$ is the group of integers and $\widehat{f}=\{\widehat{f}(k), k \in \mathbb{Z}\}$ is the sequence of Fourier coefficients of a function $f$,

$$
\widehat{f}(k)=\int_{\mathbb{T}} f(t) e^{-i k t} \frac{d t}{2 \pi}, \quad k \in \mathbb{Z} .
$$

The class $A(\mathbb{T})$ is a Banach space with respect to the natural norm

$$
\|f\|_{A(\mathbb{T})}=\|\widehat{f}\|_{l^{1}(\mathbb{Z})}=\sum_{k \in \mathbb{Z}}|\widehat{f}(k)|
$$

It is well known that $A(\mathbb{T})$ is a Banach algebra (with the usual multiplication of functions). 
We identify continuous mappings $\varphi$ of the circle $\mathbb{T}$ into itself and continuous functions $\varphi: \mathbb{R} \rightarrow \mathbb{R}$ satisfying

$$
\varphi(t+2 \pi)=\varphi(t)(\bmod 2 \pi) .
$$

Recall the known Beurling-Helson theorem [1] (see also [2], [3]). Let $\varphi: \mathbb{T} \rightarrow \mathbb{T}$ be a continuous mapping such that

$$
\left\|e^{i n \varphi}\right\|_{A(\mathbb{T})}=O(1), \quad n \in \mathbb{Z}
$$

then $\varphi$ is linear (with integer slope) i.e. $\varphi(t)=\nu t+\varphi(0)$, where $\nu \in \mathbb{Z}$. This theorem immediately implies the solution of the Levy problem on the description of endomorphisms of the algebra $A(\mathbb{T})$ : all these endomorphisms are trivial, i.e., have the form $f(t) \rightarrow f\left(\nu t+t_{0}\right)$. In other words only trivial changes of variable are admissible in $A(\mathbb{T})$. Note also another version of the Beurling-Helson theorem: if $U$ is a bounded translation invariant operator from $l^{1}(\mathbb{Z})$ to itself such that $\left\|U^{n}\right\|_{l^{1} \rightarrow l^{1}}=O(1), n \in \mathbb{Z}$, then $U=\xi S$, where $\xi$ is a complex number, $|\xi|=1$, and $S$ is a translation.

The character of the growth of the norms $\left\|e^{i n \varphi}\right\|_{A(\mathbb{T})}$ is in general not clear. We shall briefly indicate certain known results. It is easy to show (see $\left[2\right.$, Ch. VI, § 3]) that for every $C^{1}$-smooth mapping $\varphi$ we have $\left\|e^{i n \varphi}\right\|_{A(\mathbb{T})}=$ $O(\sqrt{|n|})$. At the same time if a mapping $\varphi$ is $C^{2}$-smooth and nonlinear then

$$
\left\|e^{i n \varphi}\right\|_{A(\mathbb{T})} \geq c \sqrt{|n|}, \quad n \in \mathbb{Z},
$$

where $c=c(\varphi)$ is independent of $n$. This result is contained implicitly in Z. L. Leibenson's work [4] and was obtained in explicit form by J. -P. Kahane [5] with the use of Leibenson's approach (for a simple proof see [2, Ch. VI, $\S 3])$. Thus, for every nonlinear $C^{2}$-smooth mapping $\varphi$ we have

$$
\left\|e^{i n \varphi}\right\|_{A(\mathbb{T})} \simeq \sqrt{|n|}, \quad|n| \rightarrow \infty .
$$

The growth of the norms $\left\|e^{i n \varphi}\right\|_{A(\mathbb{T})}$ in the $C^{1}$-smooth case was studied by the author of the present paper in [6], [7] (see also [8] where we considered functions of several variables). Note that the $C^{1}$-smooth case is essentially different from the $C^{2}$-smooth case.

In general, the norms $\left\|e^{i n \varphi}\right\|_{A(\mathbb{T})}$ can grow rather slowly. Kahane [2, Ch. VI, $\S 2$ ] showed that if $\varphi: \mathbb{T} \rightarrow \mathbb{T}$ is a piecewise linear but not linear continuous mapping, then $\left\|e^{i n \varphi}\right\|_{A(\mathbb{T})} \simeq \log |n|$. 
It is unknown if for nonlinear continuous mappings $\varphi$ the norms $\left\|e^{i n \varphi}\right\|_{A(\mathbb{T})}$ can grow slower than $\log |n|$. Kahane conjectured that the Beurling-Helson theorem can be strengthened, in particular, he conjectured that the condition

$$
\left\|e^{i n \varphi}\right\|_{A(\mathbb{T})}=o(\log |n|), \quad|n| \rightarrow \infty,
$$

implies that $\varphi$ is linear. As far as the author knows this conjecture was first proposed by Kahane in his talk presented at the ICM 1962 in Stockholm, [9]. Later the conjecture was mentioned by Kahane in [2] and [3].1.

Here we shall obtain the following theorem.

Theorem. Let $\varphi: \mathbb{T} \rightarrow \mathbb{T}$ be a continuous mapping. Suppose that

$$
\left\|e^{i n \varphi}\right\|_{A(\mathbb{T})}=o\left(\left(\frac{\log \log |n|}{\log \log \log |n|}\right)^{1 / 12}\right), \quad n \in \mathbb{Z}, \quad|n| \rightarrow \infty .
$$

Then $\varphi$ is linear, i.e., $\varphi(t)=\nu t+\varphi(0), \nu \in \mathbb{Z}$.

Ideologically the proof of our theorem is to some extent close to the proof of the Beurling-Helson theorem given by Kahane in [3] (the proof in [3] is based on completely different approach rather than the original proof by Beurling and Helson [1], [2]). We modify Kahane's arguments so that instead of dealing with the group $\mathbb{T}$ and the mapping $\varphi$ itself we apply the arguments to the cyclic group $\mathbb{T}_{N}$ for large $N^{\prime} s$ and a certain mapping $\varphi_{N}$, which on $\mathbb{T}_{N}$ provides a good approximation for $\varphi$ and whose values are rational numbers with a "small common denominator". The construction of this mapping is based on the Dirichlet theorem on simultaneous diophantine approximation. The key role in the proof is played by the result recently obtained by B. Green and S. V. Konyagin [10, Theorem 1.3].

Before proving the theorem let us introduce notation, recall certain (quite standard, see, e.g., [11]) facts of harmonic analysis on $\mathbb{T}_{N}$, and discuss the Green-Konyagin theorem.

Let $G$ be a finite abelian group. We use the normalised counting measure $\mu_{G}$ on the group $G$ which attaches weight $1 /(\operatorname{card} G)$ to each point $x \in G$ (by card $X$ we denote the number of the elements of a finite set $X$ ). Thus, if $E \subseteq G$, then

$$
\mu_{G}(E)=\frac{\operatorname{card} E}{\operatorname{card} G}
$$

\footnotetext{
${ }^{1}$ Note that a priori the mere existence of a sequence $\omega_{n} \rightarrow \infty$ such that the condition $\left\|e^{i n \varphi}\right\|_{A(\mathbb{T})}=o\left(\omega_{|n|}\right)$ implies linearity of a mapping $\varphi$ is not obvious.
} 
and if $f$ is a (complex) function on $G$, then

$$
\int_{G} f(x) d \mu_{G}(x)=\frac{1}{\operatorname{card} G} \sum_{x \in G} f(x) .
$$

We shall often write $\int_{G} f(x) d x$ or $\int_{G} f d \mu_{G}$ instead of $\int_{G} f(x) d \mu_{G}(x)$.

We put

$$
\|f\|_{L^{2}(G)}=\left(\int_{G}|f(x)|^{2} d x\right)^{1 / 2}, \quad\|f\|_{L^{\infty}(G)}=\max _{x \in G}|f(x)| .
$$

In general, for a set $E \subseteq G$ and a function $f$ on $E$ we put

$$
\|f\|_{L^{\infty}(E)}=\max _{x \in E}|f(x)| .
$$

Any finite abelian group $G$ endowed with the measure $\mu_{G}$ is a probability space. It will be convenient to consider the general case. Let $(\mathbb{X}, \mu)$ be a probability space. For a set $E \subseteq \mathbb{X}$ we denote by $1_{E}$ its characteristic function: $1_{E}(x)=1$ if $x \in E$ and $1_{E}(x)=0$ if $x \notin E$. For an arbitrary $\mu$-measurable set $E \subseteq \mathbb{X}$ let

$$
\delta_{\mathbb{X}, \mu}(E)=\min (\mu(E), 1-\mu(E)) .
$$

Clearly we always have $0 \leq \delta_{\mathbb{X}, \mu}(E) \leq 1 / 2$. If $\delta_{\mathbb{X}, \mu}(E)$ is very small then the set $E$ is either "very small" or "very large" (i.e., its compliment is small).

We denote by $\mathbb{T}_{N}$ the cyclic group

$$
\mathbb{T}_{N}=\left\{\frac{2 \pi j}{N}, j=0,1, \ldots, N-1\right\}
$$

regarded as a subgroup of $\mathbb{T}$ (with addition $\bmod 2 \pi$ ). The characters of $\mathbb{T}_{N}$ are the functions $e_{k}, k=0,1, \ldots, N-1$, defined as follows:

$$
e_{k}\left(\frac{2 \pi j}{N}\right)=e^{i k \frac{2 \pi j}{N}}, \quad j=0,1, \ldots, N-1
$$

We realize the group dual to $\mathbb{T}_{N}$ as the group

$$
\mathbb{Z}_{N}=\{0,1, \ldots, N-1\}
$$


considered with addition mod $N$. If $f$ is a function on $\mathbb{T}_{N}$, then its Fourier transform $\widehat{f}=\left\{\widehat{f}(k), k \in \mathbb{Z}_{N}\right\}$ on the group $\mathbb{T}_{N}$ is defined by

$$
\widehat{f}(k)=\int_{\mathbb{T}_{N}} f \overline{e_{k}} d \mu_{\mathbb{T}_{N}}, \quad k \in \mathbb{Z}_{N}
$$

(the bar stands for the complex conjugation). We use the same notation $\widehat{f}$ for the Fourier transform on $\mathbb{T}$ and on $\mathbb{T}_{N}$ but this does not lead to confusion; in what follows, up to the end of the proof of the theorem, $\widehat{f}$ stands for the Fourier transform on $\mathbb{T}_{N}$.

Let $f$ be an arbitrary (complex) function on $\mathbb{T}_{N}$. We have

$$
f(t)=\sum_{k \in \mathbb{Z}_{N}} \widehat{f}(k) e_{k}(t), \quad t \in \mathbb{T}_{N}
$$

Put

$$
\|f\|_{A\left(\mathbb{T}_{N}\right)}=\|\widehat{f}\|_{l^{1}\left(\mathbb{Z}_{N}\right)}=\sum_{k \in \mathbb{Z}_{N}}|\widehat{f}(k)| .
$$

It is easy to verify that for any two functions $f_{1}, f_{2}$ on $\mathbb{T}_{N}$ we have

$$
\left\|f_{1} f_{2}\right\|_{A\left(\mathbb{T}_{N}\right)} \leq\left\|f_{1}\right\|_{A\left(\mathbb{T}_{N}\right)}\left\|f_{2}\right\|_{A\left(\mathbb{T}_{N}\right)} .
$$

Generally, for $p \geq 1$ we put

$$
\|f\|_{A_{p}\left(\mathbb{T}_{N}\right)}=\|\widehat{f}\|_{l^{p}\left(\mathbb{Z}_{N}\right)}=\left(\sum_{k \in \mathbb{Z}_{N}}|\widehat{f}(k)|^{p}\right)^{1 / p}
$$

(we write $A\left(\mathbb{T}_{N}\right)$ instead of $A_{1}\left(\mathbb{T}_{N}\right)$ ).

The Parseval identity has the form

$$
\|f\|_{L^{2}\left(\mathbb{T}_{N}\right)}=\|f\|_{A_{2}\left(\mathbb{T}_{N}\right)} .
$$

For any two functions $f_{1}, f_{2}$ on $\mathbb{T}_{N}$ we define their convolution $f_{1} * f_{2}$ by

$$
f_{1} * f_{2}(t)=\int_{\mathbb{T}_{N}} f_{1}(x) f_{2}(t-x) d x, \quad t \in \mathbb{T}_{N}
$$

We have $\widehat{f_{1} * f_{2}}=\widehat{f_{1}} \widehat{f}_{2}$.

Now we recall the Green-Konyagin theorem. Note first the trivial fact: if $E$ is a set on the circle $\mathbb{T}$ such that the Fourier transform of its characteristic 
function $1_{E}$ belongs to $l^{1}(\mathbb{Z})$, then either the set $E$ has (Lebesgue) measure zero or it is almost the whole circle $\mathbb{T}$. This effect can be viewed in more detail on the cyclic group $\mathbb{T}_{N}$. Namely, according to the Green-Konyagin theorem, if $N$ is a sufficiently large prime number, then for any real function $f$ on $\mathbb{T}_{N}$ with $\int_{\mathbb{T}_{N}} f d \mu_{\mathbb{T}_{N}}=0$ we have

$$
\min _{t \in \mathbb{T}_{N}}|f(t)| \leq c\left(\frac{\log \log N}{\log N}\right)^{1 / 3}\|f\|_{A\left(\mathbb{T}_{N}\right)},
$$

where the constant $c>0$ is independent of $N$ and $f$. Taking an arbitrary set $E \subseteq \mathbb{T}_{N}$ and applying this estimate to $f(t)=1_{E}(t)-\mu_{\mathbb{T}_{N}}(E)$, we see that

$$
\delta_{\mathbb{T}_{N}, \mu_{\mathbb{T}_{N}}}(E) \leq c\left(\frac{\log \log N}{\log N}\right)^{1 / 3}\left\|1_{E}\right\|_{A\left(\mathbb{T}_{N}\right)},
$$

where $c>0$ is independent of $N$ and $E$. (We took into account that $\mu_{\mathbb{T}_{N}}(E)=\widehat{1_{E}}(0)$ whence $\|f\|_{A\left(\mathbb{T}_{N}\right)} \leq\left\|1_{E}\right\|_{A\left(\mathbb{T}_{N}\right)}$.)

We shall also need the Dirichlet theorem on simultaneous diophantine approximation (see, e.g., [12, Ch. II, $\S 1]$ ). If $\alpha_{1}, \alpha_{2}, \ldots, \alpha_{N}$ are real numbers and $D \geq 1$ is an integer, then there exist integers $Q, P_{1}, P_{2}, \ldots, P_{N}$ such that $1 \leq Q \leq D^{N}$ and

$$
\left|\alpha_{j} Q-P_{j}\right| \leq \frac{1}{D}, \quad j=1,2, \ldots, N
$$

Proof of the Theorem. Since the mapping $\varphi$ is continuous, we have

$$
\varphi(t+2 \pi)=\varphi(t)+2 \pi k
$$

where $k \in \mathbb{Z}$ is independent of $t$. Replacing the function $\varphi$ by $\varphi(t)-k t$, we can assume that

$$
\varphi(t+2 \pi)=\varphi(t)
$$

i.e., that $\varphi$ is a real continuous function on the circle $\mathbb{T}$.

Note also that just by the assumption of the theorem we have $e^{i n \varphi} \in$ $A(\mathbb{T})$ for all sufficiently large $n \in \mathbb{Z}$. Taking sufficiently large $n$ we have $e^{i n \varphi} \in A(\mathbb{T})$ and $e^{i(n+1) \varphi} \in A(\mathbb{T})$. Then $e^{-i n \varphi}=\overline{e^{i n \varphi}} \in A(\mathbb{T})$, and we see that $e^{i \varphi}=e^{i(n+1) \varphi} e^{-i n \varphi} \in A(\mathbb{T})$. Therefore, $e^{i n \varphi} \in A(\mathbb{T})$ for all $n \in \mathbb{Z}$.

We put

$$
\Theta(n)=\max _{\lambda=0,1,2, \ldots, n}\left\|e^{i \lambda \varphi}\right\|_{A(\mathbb{T})} .
$$


The sequence $\Theta(n), n=0,1,2, \ldots$, is non-decreasing, $\Theta(n) \geq \Theta(0)=1$ for all $n$, and, by the assumption of the theorem,

$$
\Theta(n)=o\left(\left(\frac{\log \log n}{\log \log \log n}\right)^{1 / 12}\right)
$$

We have

$$
\left\|e^{i n \varphi}\right\|_{A(\mathbb{T})} \leq \Theta(n), \quad n=0,1,2, \ldots,
$$

Everywhere below in the proof up to Lemma 4 we assume that $N$ is an arbitrary positive integer.

Fixing $N$ and applying the Dirichlet theorem to the numbers

$$
\alpha_{j}=\frac{1}{2 \pi} \varphi\left(\frac{2 \pi j}{N}\right), \quad j=0,1, \ldots, N-1,
$$

and $D=N$, we find integers $Q_{N}$ and $P_{N, j}, j=0,1, \ldots, N-1$, such that

$$
1 \leq Q_{N} \leq N^{N}
$$

and

$$
\left|\frac{1}{2 \pi} \varphi\left(\frac{2 \pi j}{N}\right) Q_{N}-P_{N, j}\right| \leq \frac{1}{N}, \quad j=0,1, \ldots, N-1 .
$$

Let us define a function $\varphi_{N}$ on $\mathbb{T}_{N}$ by

$$
\varphi_{N}\left(\frac{2 \pi j}{N}\right)=\frac{2 \pi P_{N, j}}{Q_{N}}, \quad j=0,1, \ldots, N-1 .
$$

We see that

$$
\left|\varphi\left(\frac{2 \pi j}{N}\right)-\varphi_{N}\left(\frac{2 \pi j}{N}\right)\right| \leq \frac{2 \pi}{N Q_{N}}, \quad j=0,1, \ldots, N-1 .
$$

That is

$$
\left\|\varphi-\varphi_{N}\right\|_{L^{\infty}\left(\mathbb{T}_{N}\right)} \leq \frac{2 \pi}{N Q_{N}}
$$

Lemma 1. For all $n=0,1,2, \ldots, Q_{N}-1$ we have $\left\|e^{i n \varphi_{N}}\right\|_{A\left(\mathbb{T}_{N}\right)} \leq$ $8 \Theta\left(N^{N}\right)$. 
Proof. Let $f$ be an arbitrary function in $A(\mathbb{T})$. We have

$$
f(t)=\sum_{k \in \mathbb{Z}} c_{k} e^{i k t}, \quad \sum_{k \in \mathbb{Z}}\left|c_{k}\right|=\|f\|_{A(\mathbb{T})}<\infty .
$$

Considering $f$ as a function on $\mathbb{T}_{N}$ and calculating its Fourier transform $\widehat{f}$ on $\mathbb{T}_{N}$, we obtain

$$
\begin{aligned}
\widehat{f}(k)=\int_{\mathbb{T}_{N}}\left(\sum_{\nu \in \mathbb{Z}} c_{\nu} e^{i \nu t}\right) e^{-i k t} d \mu_{\mathbb{T}_{N}}(t) & \\
=\sum_{\nu \in \mathbb{Z}} c_{\nu} \int_{\mathbb{T}_{N}} e^{i \nu t} e^{-i k t} d \mu_{\mathbb{T}_{N}}(t) & =\sum_{\nu=k(\bmod N)} c_{\nu}, \quad k \in \mathbb{Z}_{N},
\end{aligned}
$$

therefore,

$$
\|f\|_{A\left(\mathbb{T}_{N}\right)} \leq\|f\|_{A(\mathbb{T})} .
$$

Using this inequality, we see that (see (4))

$$
\left\|e^{i n \varphi}\right\|_{A\left(\mathbb{T}_{N}\right)} \leq \Theta(n), \quad n=0,1,2, \ldots
$$

Note that estimate (7) yields (for $n \geq 0$ )

$$
\left\|e^{i n \varphi}-e^{i n \varphi_{N}}\right\|_{L^{\infty}\left(\mathbb{T}_{N}\right)} \leq n\left\|\varphi-\varphi_{N}\right\|_{L^{\infty}\left(\mathbb{T}_{N}\right)} \leq n \frac{2 \pi}{N Q_{N}},
$$

whence for $n=0,1, \ldots, Q_{N}-1$ we obtain

$$
\left\|e^{i n \varphi}-e^{i n \varphi_{N}}\right\|_{L^{\infty}\left(\mathbb{T}_{N}\right)} \leq \frac{2 \pi}{N} .
$$

Clearly, for each function $f$ on $\mathbb{T}_{N}$ we have

$$
|\widehat{f}(k)| \leq\|f\|_{L^{\infty}\left(\mathbb{T}_{N}\right)}, \quad k \in \mathbb{Z}_{N},
$$

( $\widehat{f}$ is the Fourier transform on $\mathbb{T}_{N}$ ) whence, taking into account that card $\mathbb{Z}_{N}=$ $N$, we see that

$$
\|f\|_{A\left(\mathbb{T}_{N}\right)}=\sum_{k \in \mathbb{Z}_{N}}|\widehat{f}(k)| \leq N\|f\|_{L^{\infty}\left(\mathbb{T}_{N}\right)} .
$$

Therefore (see (9)),

$$
\left\|e^{i n \varphi}-e^{i n \varphi_{N}}\right\|_{A\left(\mathbb{T}_{N}\right)} \leq 2 \pi, \quad n=0,1, \ldots, Q_{N}-1 .
$$


Thus, for $n=0,1, \ldots, Q_{N}-1$ we obtain (see (8), (10) and (5))

$$
\begin{aligned}
&\left\|e^{i n \varphi_{N}}\right\|_{A\left(\mathbb{T}_{N}\right)} \leq\left\|e^{i n \varphi}\right\|_{A\left(\mathbb{T}_{N}\right)}+\left\|e^{i n \varphi}-e^{i n \varphi_{N}}\right\|_{A\left(\mathbb{T}_{N}\right)} \\
& \leq \Theta(n)+2 \pi \leq 8 \Theta(n) \leq 8 \Theta\left(Q_{N}\right) \leq 8 \Theta\left(N^{N}\right) .
\end{aligned}
$$

The lemma is proved.

We define a function $\Phi$ on $\mathbb{T}^{3}=\mathbb{T} \times \mathbb{T} \times \mathbb{T}$ by

$$
\Phi(x, y, z)=\varphi(x)+\varphi(z-x)-\varphi(y)-\varphi(z-y), \quad x, y, z \in \mathbb{T} .
$$

We also define a function $\Phi_{N}$ on the group $\mathbb{T}_{N}^{3}=\mathbb{T}_{N} \times \mathbb{T}_{N} \times \mathbb{T}_{N}$, by

$$
\Phi_{N}(x, y, z)=\varphi_{N}(x)+\varphi_{N}(z-x)-\varphi_{N}(y)-\varphi_{N}(z-y), \quad x, y, z \in \mathbb{T}_{N}
$$

Consider the set

$$
E_{N}=\left\{(x, y, z) \in \mathbb{T}_{N}^{3}: e^{i \Phi_{N}(x, y, z)}=1\right\} .
$$

Note that each value of the function $\varphi_{N}$ and thus each value of the function $\Phi_{N}$ as well is of the form $2 \pi P / Q_{N}$ for a certain $P \in \mathbb{Z}$ (see (6)), so the following identity holds:

$$
\frac{1}{Q_{N}} \sum_{n=0}^{Q_{N}-1} e^{i n \Phi_{N}}=1_{E_{N}}
$$

In the following lemma we establish the lower bound for the measure of the set $E_{N}$.

Lemma 2. We have

$$
\frac{1}{64\left(\Theta\left(N^{N}\right)\right)^{2}} \leq \mu_{\mathbb{T}_{N}^{3}}\left(E_{N}\right)
$$

Proof. First let us verify that if $f$ is an arbitrary real function on $\mathbb{T}_{N}$, then

$$
\frac{1}{\left\|e^{i n f}\right\|_{A\left(\mathbb{T}_{N}\right)}^{2}} \leq \int_{\mathbb{T}_{N}^{3}} e^{i n F} d \mu_{\mathbb{T}_{N}^{3}}, \quad n \in \mathbb{Z}
$$

where

$$
F(x, y, z)=f(x)+f(z-x)-f(y)-f(z-y), \quad x, y, z \in \mathbb{T}_{N}
$$


To see this we repeat word for word Kahane's arguments [3] just replacing $\mathbb{T}$ with $\mathbb{T}_{N}$. Namely, interpolating $l^{2}$ between $l^{1}$ and $l^{4}$, we obtain $\|\cdot\|_{l^{2}} \leq$ $\|\cdot\|_{l^{1}}^{1 / 3}\|\cdot\|_{l^{4}}^{2 / 3}$. Therefore,

$$
1=\left\|e^{i n f}\right\|_{L^{2}\left(\mathbb{T}_{N}\right)}=\left\|e^{i n f}\right\|_{A_{2}\left(\mathbb{T}_{N}\right)} \leq\left\|e^{i n f}\right\|_{A\left(\mathbb{T}_{N}\right)}^{1 / 3}\left\|e^{i n f}\right\|_{A_{4}\left(\mathbb{T}_{N}\right)}^{2 / 3} .
$$

Hence

$$
\begin{aligned}
\frac{1}{\left\|e^{i n f}\right\|_{A\left(\mathbb{T}_{N}\right)}^{2}} \leq\left\|e^{i n f}\right\|_{A_{4}\left(\mathbb{T}_{N}\right)}^{4}=\sum_{k \in \mathbb{Z}_{N}} \mid e^{\left.i n f * e^{i n f}(k)\right|^{2}} \\
=\left\|e^{i n f} * e^{i n f}\right\|_{L^{2}\left(\mathbb{T}_{N}\right)}^{2}=\int_{\mathbb{T}_{N}}\left|\int_{\mathbb{T}_{N}} e^{i n f(x)} e^{i n f(z-x)} d x\right|^{2} d z \\
=\int_{\mathbb{T}_{N}}\left(\int_{\mathbb{T}_{N}} e^{i n f(x)} e^{i n f(z-x)} d x\right) \overline{\left(\int_{\mathbb{T}_{N}} e^{i n f(y)} e^{i n f(z-y)} d y\right)} d z \\
=\iiint_{\mathbb{T}_{N}^{3}} e^{i n F(x, y, z)} d x d y d z .
\end{aligned}
$$

Thus we have (12).

Now we use inequality (12) for $f=\varphi_{N}$. Applying Lemma 1 , we see that for all $n=0,1, \ldots, Q_{N}-1$

$$
\frac{1}{64\left(\Theta\left(N^{N}\right)\right)^{2}} \leq \int_{\mathbb{T}_{N}^{3}} e^{i n \Phi_{N}} d \mu_{\mathbb{T}_{N}^{3}} .
$$

Therefore,

$$
\frac{1}{64\left(\Theta\left(N^{N}\right)\right)^{2}} \leq \int_{\mathbb{T}_{N}^{3}}\left(\frac{1}{Q_{N}} \sum_{n=0}^{Q_{N}-1} e^{i n \Phi_{N}}\right) d \mu_{\mathbb{T}_{N}^{3}} .
$$

It remains to use identity (11). The lemma is proved.

Our next aim is an upper bound for $\delta_{\mathbb{T}_{N}^{3}, \mu_{\mathbb{T}_{N}^{3}}}\left(E_{N}\right)$. We shall obtain it in Lemma 4 . First we prove Lemma 3 which has a technical character.

Let $\left(\mathbb{X}_{j}, \mu_{j}\right), j=1,2, \ldots m$, be probability spaces. let $(\mathbb{X}, \mu)$ be their product:

$$
\mathbb{X}=\mathbb{X}_{1} \times \mathbb{X}_{2} \times \ldots \times \mathbb{X}_{m}, \quad \mu=\mu_{1} \otimes \mu_{2} \otimes \ldots \otimes \mu_{m} .
$$

Consider a set $E \subseteq \mathbb{X}$. For each fixed $j=1,2, \ldots, m$ every point

$$
\left(x_{1}, x_{2}, \ldots, x_{j-1}, x_{j+1}, \ldots, x_{m}\right)
$$


in $\mathbb{X}_{1} \times \mathbb{X}_{2} \times \ldots \times \mathbb{X}_{j-1} \times \mathbb{X}_{j+1} \times \ldots \times \mathbb{X}_{m}$ defines a $j$-section of $E$, namely, the set

$$
E^{x_{1}, x_{2}, \ldots, x_{j-1}, x_{j+1}, \ldots, x_{m}}=\left\{x_{j} \in \mathbb{X}_{j}:\left(x_{1}, x_{2}, \ldots, x_{j-1}, x_{j}, x_{j+1}, \ldots, x_{m}\right) \in E\right\} .
$$

Lemma 3. Let $\left(\mathbb{X}_{j}, \mu_{j}\right), j=1,2, \ldots, m$, be probability spaces and $(\mathbb{X}, \mu)$ their product. Let $E$ be a $\mu$-measurable set in $\mathbb{X}$. Let $\delta \geq 0$. Suppose that for each $j=1,2, \ldots, m$ every $j$-section $E^{x_{1}, x_{2}, \ldots, x_{j-1}, x_{j+1}, \ldots, x_{m}}$ of $E$ satisfies the condition that $\delta_{\mathbb{X}_{j}, \mu_{j}}\left(E^{x_{1}, x_{2}, \ldots, x_{j-1}, x_{j+1}, \ldots, x_{m}}\right) \leq \delta$. Then $\delta_{\mathbb{X}, \mu}(E) \leq 3^{m-1} \delta$.

Proof 2 First we shall show that the assertion of the lemma is true for $m=2$. Let $\left(\mathbb{X}_{1}, \mu_{1}\right)$ and $\left(\mathbb{X}_{2}, \mu_{2}\right)$ be probability spaces. Put $\mathbb{X}=\mathbb{X}_{1} \times \mathbb{X}_{2}$ and $\mu=\mu_{1} \otimes \mu_{2}$. Consider an arbitrary $\mu$-measurable set $E \subseteq \mathbb{X}$ whose every 1 -section

$$
E^{x_{2}}=\left\{x_{1} \in \mathbb{X}_{1}:\left(x_{1}, x_{2}\right) \in E\right\}
$$

and every 2 -section

$$
E^{x_{1}}=\left\{x_{2} \in \mathbb{X}_{2}:\left(x_{1}, x_{2}\right) \in E\right\}
$$

satisfy

$$
\delta_{\mathbb{X}_{1}, \mu_{1}}\left(E^{x_{2}}\right) \leq \delta
$$

and correspondingly

$$
\delta_{\mathbb{X}_{2}, \mu_{2}}\left(E^{x_{1}}\right) \leq \delta
$$

Let us verify that $\delta_{\mathbb{X}, \mu}(E) \leq 3 \delta$.

We put

$$
\mathbb{X}_{1}^{<}=\left\{x_{1} \in \mathbb{X}_{1}: \mu_{2}\left(E^{x_{1}}\right) \leq \delta\right\}, \quad \mathbb{X}_{1}^{>}=\left\{x_{1} \in \mathbb{X}_{1}: \mu_{2}\left(E^{x_{1}}\right) \geq 1-\delta\right\},
$$

and similarly

$$
\mathbb{X}_{2}^{<}=\left\{x_{2} \in \mathbb{X}_{2}: \mu_{1}\left(E^{x_{2}}\right) \leq \delta\right\}, \quad \mathbb{X}_{2}^{>}=\left\{x_{2} \in \mathbb{X}_{2}: \mu_{1}\left(E^{x_{2}}\right) \geq 1-\delta\right\}
$$

We can assume that $0 \leq \delta<1 / 2$ (for $\delta \geq 1 / 2$ the assertion of the lemma is trivial). Then

$$
\mathbb{X}_{j}^{<} \cap \mathbb{X}_{j}^{>}=\varnothing, \quad \mathbb{X}_{j}^{<} \cup \mathbb{X}_{j}^{>}=\mathbb{X}_{j}, \quad j=1,2
$$

\footnotetext{
${ }^{2}$ Perhaps the assertion of Lemma 3 (in this form or another) is known, but the author has not been able to find an appropriate reference.
} 
We put

$$
\alpha_{1}=\mu_{1}\left(\mathbb{X}_{1}^{>}\right), \quad \alpha_{2}=\mu_{2}\left(\mathbb{X}_{2}^{>}\right)
$$

Note that

$$
\begin{gathered}
\iint_{\mathbb{X}_{1}^{>} \times \mathbb{X}_{2}^{<}} 1_{E}\left(x_{1}, x_{2}\right) d \mu_{1}\left(x_{1}\right) d \mu_{2}\left(x_{2}\right) \leq \iint_{\mathbb{X}_{1} \times \mathbb{X}_{2}^{<}} 1_{E}\left(x_{1}, x_{2}\right) d \mu_{1}\left(x_{1}\right) d \mu_{2}\left(x_{2}\right) \\
=\int_{\mathbb{X}_{2}^{<}} \mu_{1}\left(E^{x_{2}}\right) d \mu_{2}\left(x_{2}\right) \leq \delta \mu_{2}\left(X_{2}^{<}\right)=\delta\left(1-\alpha_{2}\right)
\end{gathered}
$$

At the same time it is obvious that

$$
\iint_{\mathbb{X}_{1}^{>} \times \mathbb{X}_{2}^{>}} 1_{E}\left(x_{1}, x_{2}\right) d \mu_{1}\left(x_{1}\right) d \mu_{2}\left(x_{2}\right) \leq \mu_{1}\left(\mathbb{X}_{1}^{>}\right) \mu_{2}\left(\mathbb{X}_{2}^{>}\right)=\alpha_{1} \alpha_{2}
$$

Adding (13) and (14), we obtain

$$
\iint_{\mathbb{X}_{1}^{>} \times \mathbb{X}_{2}} 1_{E}\left(x_{1}, x_{2}\right) d \mu_{1}\left(x_{1}\right) d \mu_{2}\left(x_{2}\right) \leq \delta\left(1-\alpha_{2}\right)+\alpha_{1} \alpha_{2} .
$$

On the other hand

$$
\begin{gathered}
\iint_{\mathbb{X}_{1}^{>} \times \mathbb{X}_{2}} 1_{E}\left(x_{1}, x_{2}\right) d \mu_{1}\left(x_{1}\right) d \mu_{2}\left(x_{2}\right)=\int_{\mathbb{X}_{1}^{>}} \mu_{2}\left(E^{x_{1}}\right) d \mu_{1}\left(x_{1}\right) \\
\geq(1-\delta) \mu_{1}\left(\mathbb{X}_{1}^{>}\right)=(1-\delta) \alpha_{1}
\end{gathered}
$$

Together inequalities (15), (16) yield

$$
(1-\delta) \alpha_{1} \leq \delta\left(1-\alpha_{2}\right)+\alpha_{1} \alpha_{2}
$$

Similarly

$$
(1-\delta) \alpha_{2} \leq \delta\left(1-\alpha_{1}\right)+\alpha_{2} \alpha_{1}
$$

Adding inequalities (17) and (18), we see that

$$
\alpha_{1}+\alpha_{2} \leq 2 \delta+2 \alpha_{1} \alpha_{2}
$$

that is

$$
\alpha_{1}\left(1-\alpha_{2}\right)+\alpha_{2}\left(1-\alpha_{1}\right) \leq 2 \delta .
$$

Hence, putting $a=\min \left(\alpha_{1}, 1-\alpha_{1}\right)$, we have

$$
a=a\left(1-\alpha_{2}\right)+\alpha_{2} a \leq \alpha_{1}\left(1-\alpha_{2}\right)+\alpha_{2}\left(1-\alpha_{1}\right) \leq 2 \delta .
$$


Thus, only two cases are possible: either $\alpha_{1} \leq 2 \delta$ or $\alpha_{1} \geq 1-2 \delta$.

In the first case we obtain

$$
\begin{aligned}
& \mu(E)=\int_{\mathbb{X}_{1}} \mu_{2}\left(E^{x_{1}}\right) d \mu_{1}\left(x_{1}\right) \\
& =\int_{\mathbb{X}_{1}^{>}} \mu_{2}\left(E^{x_{1}}\right) d \mu_{1}\left(x_{1}\right)+\int_{\mathbb{X}_{1}^{<}} \mu_{2}\left(E^{x_{1}}\right) d \mu_{1}\left(x_{1}\right) \\
& \quad \leq \mu_{1}\left(\mathbb{X}_{1}^{>}\right)+\delta \mu_{1}\left(\mathbb{X}_{1}^{<}\right)=\alpha_{1}+\delta\left(1-\alpha_{1}\right) \leq \alpha_{1}+\delta \leq 3 \delta .
\end{aligned}
$$

In the second case we obtain

$$
\begin{aligned}
\mu(E)=\int_{\mathbb{X}_{1}} \mu_{2}\left(E^{x_{1}}\right) d \mu_{1}\left(x_{1}\right) \geq \int_{\mathbb{X}_{1}^{>}} \mu_{2}\left(E^{x_{1}}\right) d \mu_{1}\left(x_{1}\right) \geq(1-\delta) \mu_{1}\left(\mathbb{X}_{1}^{>}\right) \\
=(1-\delta) \alpha_{1} \geq(1-\delta)(1-2 \delta)=1-3 \delta+2 \delta^{2} \geq 1-3 \delta .
\end{aligned}
$$

Thus, the assertion of the lemma is true for $m=2$.

For an arbitrary $m$ the assertion of the lemma easily follows by induction. Assume that the assertion of the lemma holds for a certain $m \geq 2$. Let $\left(\mathbb{X}_{j}, \mu_{j}\right), j=1,2, \ldots, m+1$, be probability spaces. It suffices to consider two spaces, one of which is $\mathbb{X}_{1} \times \mathbb{X}_{2} \times \ldots \times \mathbb{X}_{m}$, with the measure $\mu_{1} \otimes \mu_{2} \otimes \ldots \otimes \mu_{m}$, and the other one is $\mathbb{X}_{m+1}$ with the measure $\mu_{m+1}$. The lemma is proved.

Lemma 4. If $N$ is a sufficiently large prime number, then

$$
\delta_{\mathbb{T}_{N}^{3}, \mu_{\mathbb{T}_{N}^{3}}}\left(E_{N}\right) \leq c\left(\frac{\log \log N}{\log N}\right)^{1 / 3}\left(\Theta\left(N^{N}\right)\right)^{2},
$$

where $c>0$ is independent of $N$.

Proof. Fix arbitrary $y \in \mathbb{T}_{N}$ and $z \in \mathbb{T}_{N}$. Consider the corresponding 1 -section of the set $E_{N}$, namely, the set

$$
E_{N}^{y, z}=\left\{x \in \mathbb{T}_{N}:(x, y, z) \in E_{N}\right\} .
$$

Consider also the corresponding section $\Phi_{N}^{y, z}$ of the function $\Phi_{N}$, i.e., the function of $x \in \mathbb{T}_{N}$, defined as follows:

$$
\Phi_{N}^{y, z}(x)=\Phi_{N}(x, y, z) .
$$


Note that in the product

$$
e^{i n \Phi_{N}^{y, z}(x)}=e^{i n \varphi_{N}(x)} e^{i n \varphi_{N}(z-x)} e^{-i n \varphi_{N}(y)} e^{-i n \varphi_{N}(z-y)}
$$

only two factors are functions of $x$ both having $A\left(\mathbb{T}_{N}\right)$ norm equal to $\left\|e^{i n \varphi_{N}}\right\|_{A\left(\mathbb{T}_{N}\right)}$ while the two remaining factors are constants of modulus 1. So,

$$
\left\|e^{i n \Phi_{N}^{y, z}}\right\|_{A\left(\mathbb{T}_{N}\right)} \leq\left\|e^{i n \varphi_{N}}\right\|_{A\left(\mathbb{T}_{N}\right)}^{2} .
$$

Hence, using Lemma 1, we see that

$$
\left\|e^{i n \Phi_{N}^{y, z}}\right\|_{A\left(\mathbb{T}_{N}\right)} \leq\left(8 \Theta\left(N^{N}\right)\right)^{2}, \quad n=0,1,2, \ldots, Q_{N}-1
$$

At the same time, due to identity (11), for the section $E_{N}^{y, z}$ of the set $E_{N}$ we have

$$
1_{E_{N}^{y, z}}=\frac{1}{Q_{N}} \sum_{n=0}^{Q_{N}-1} e^{i n \Phi_{N}^{y, z}}
$$

Therefore,

$$
\left\|1_{E_{N}^{y, z}}\right\|_{A\left(\mathbb{T}_{N}\right)} \leq\left(8 \Theta\left(N^{N}\right)\right)^{2} .
$$

Hence, using estimate (2), we see that for an arbitrary 1 -section $E_{N}^{y, z}$ of the set $E_{N}$ we have

$$
\delta_{\mathbb{T}_{N}, \mu_{\mathbb{T}_{N}}}\left(E_{N}^{y, z}\right) \leq c\left(\frac{\log \log N}{\log N}\right)^{1 / 3}\left(8 \Theta\left(N^{N}\right)\right)^{2},
$$

where $c>0$ is independent of $y, z$ and $N$.

In the same way we obtain similar upper estimate for an arbitrary 2 -section

$$
E_{N}^{x, z}=\left\{y \in \mathbb{T}_{N}:(x, y, z) \in E_{N}\right\}
$$

and an arbitrary 3 -section

$$
E_{N}^{x, y}=\left\{z \in \mathbb{T}_{N}:(x, y, z) \in E_{N}\right\}
$$

Using Lemma 3 for $m=3$ and $\left(\mathbb{X}_{j}, \mu_{j}\right)=\left(\mathbb{T}_{N}, \mu_{\mathbb{T}_{N}}\right), j=1,2,3$, we see that

$$
\delta_{\mathbb{T}_{N}^{3}, \mu_{\mathbb{T}_{N}^{3}}}\left(E_{N}\right) \leq 9 c\left(\frac{\log \log N}{\log N}\right)^{1 / 3}\left(8 \Theta\left(N^{N}\right)\right)^{2} .
$$

The lemma is proved. 
Consider the compliment $F_{N}=\mathbb{T}_{N}^{3} \backslash E_{N}$ of the set $E_{N}$.

Lemma 5. We have $\mu_{\mathbb{T}_{N}^{3}}\left(F_{N}\right) \rightarrow 0$ as $N$ tends to $\infty$ running over the set of prime numbers.

Proof. If $N$ is a sufficiently large prime number, then by Lemma 4 we have either

or

$$
\mu_{\mathbb{T}_{N}^{3}}\left(E_{N}\right) \leq c\left(\frac{\log \log N}{\log N}\right)^{1 / 3}\left(\Theta\left(N^{N}\right)\right)^{2}
$$

$$
\mu_{\mathbb{T}_{N}^{3}}\left(F_{N}\right) \leq c\left(\frac{\log \log N}{\log N}\right)^{1 / 3}\left(\Theta\left(N^{N}\right)\right)^{2} .
$$

In the first case, due to Lemma 2, we obtain

$$
\frac{1}{64\left(\Theta\left(N^{N}\right)\right)^{2}} \leq c\left(\frac{\log \log N}{\log N}\right)^{1 / 3}\left(\Theta\left(N^{N}\right)\right)^{2},
$$

that is

$$
\frac{1}{64 c}\left(\frac{\log N}{\log \log N}\right)^{1 / 3} \leq\left(\Theta\left(N^{N}\right)\right)^{4},
$$

which is impossible if $N$ is too large (see (3)). Thus, for all sufficiently large prime $N$ we have estimate (19), whence, taking (3) into account, we obtain

$$
\mu_{\mathbb{T}_{N}^{3}}\left(F_{N}\right) \leq c\left(\frac{\log \log N}{\log N}\right)^{1 / 3} o\left(\left(\frac{\log N}{\log \log N}\right)^{1 / 6}\right)=o(1) .
$$

The lemma is proved.

Now we shall conclude the proof of the theorem. Note that (since $e^{i \Phi_{N}}=$ 1 on $E_{N}$ ) estimate (7) yields

$$
\begin{aligned}
& \left\|e^{i \Phi}-1\right\|_{L^{\infty}\left(E_{N}\right)}=\left\|e^{i \Phi}-e^{i \Phi_{N}}\right\|_{L^{\infty}\left(E_{N}\right)} \leq\left\|\Phi-\Phi_{N}\right\|_{L^{\infty}\left(E_{N}\right)} \\
\leq & \left\|\Phi-\Phi_{N}\right\|_{L^{\infty}\left(\mathbb{T}_{N}^{3}\right)} \leq 4\left\|\varphi-\varphi_{N}\right\|_{L^{\infty}\left(\mathbb{T}_{N}\right)} \leq \frac{8 \pi}{N Q_{N}} \leq \frac{8 \pi}{N}=o(1), \quad N \rightarrow \infty .
\end{aligned}
$$


Hence, using Lemma 5, we see that as $N$ tends to infinity running over prime numbers, we have

$$
\begin{aligned}
\int_{\mathbb{T}_{N}^{3}}\left|e^{i \Phi}-1\right| d \mu_{\mathbb{T}_{N}^{3}}=\int_{E_{N}}\left|e^{i \Phi}-1\right| d \mu_{\mathbb{T}_{N}^{3}}+\int_{F_{N}}\left|e^{i \Phi}-1\right| d \mu_{\mathbb{T}_{N}^{3}} \\
\leq\left\|e^{i \Phi}-1\right\|_{L^{\infty}\left(E_{N}\right)}+2 \mu_{\mathbb{T}_{N}^{3}}\left(F_{N}\right) \rightarrow 0 .
\end{aligned}
$$

At the same time (since the function $\Phi$ is continuous on $\mathbb{T}^{3}$ ), we have

$$
\begin{aligned}
& \int_{\mathbb{T}_{N}^{3}}\left|e^{i \Phi}-1\right| d \mu_{\mathbb{T}_{N}^{3}} \\
& =\frac{1}{N^{3}} \sum_{0 \leq k, l, m \leq N-1}\left|e^{i \Phi\left(\frac{2 \pi k}{N}, \frac{2 \pi l}{N}, \frac{2 \pi m}{N}\right)}-1\right| \rightarrow \frac{1}{(2 \pi)^{3}} \int_{\mathbb{T}^{3}}\left|e^{i \Phi(x, y, z)}-1\right| d x d y d z
\end{aligned}
$$

as $N \rightarrow \infty$. Thus,

$$
\int_{\mathbb{T}^{3}}\left|e^{i \Phi(x, y, z)}-1\right| d x d y d z=0 .
$$

Hence (since $\Phi$ is continuous) we obtain

$$
e^{i \Phi(x, y, z)}=1
$$

for all $(x, y, z) \in \mathbb{T}^{3}$ and we see that

$$
\Phi(x, y, z)=2 \pi k, \quad(x, y, z) \in \mathbb{T}^{3},
$$

where $k$ is an integer independent of $(x, y, z)$. Taking $(x, y, z)=(0,0,0)$ we obtain $k=0$, whence $\Phi(x, y, z)=0$ on $\mathbb{T}^{3}$. That is

$$
\varphi(x)+\varphi(z-x)-\varphi(y)-\varphi(z-y)=0
$$

for all $x, y, z \in \mathbb{R}$. The assertion of the theorem follows.

\section{Remarks.}

1. The theorem obtained in this work has the following (equivalent) operator version. Let $U$ be a bounded translation invariant operator from $l^{1}(\mathbb{Z})$ to itself such that

$$
\left\|U^{n}\right\|_{l^{1} \rightarrow l^{1}}=o\left(\left(\frac{\log \log |n|}{\log \log \log |n|}\right)^{1 / 12}\right), \quad|n| \rightarrow \infty, \quad n \in \mathbb{Z},
$$


then $U=\xi S$, where $\xi$ is a complex number, $|\xi|=1$, and $S$ is a translation. Indeed, it is easy to verify (and is well known, see, e.g., [13]) that each bounded translation invariant operator from $l^{1}(\mathbb{Z})$ to itself is an operator of convolution with a certain sequence that belongs to $l^{1}(\mathbb{Z})$ and the norm of the operator is equal to the $l^{1}$ norm of the sequence. In particular, $U$ is an operator of convolution with a certain sequence $\left\{u_{k}, k \in \mathbb{Z}\right\} \in l^{1}$. Define a function $u$ on $\mathbb{T}$ by

$$
u(t)=\sum_{k \in \mathbb{Z}} u_{k} e^{i k t}
$$

We have $u \in A(\mathbb{T})$ and

$$
\left\|u^{n}\right\|_{A(\mathbb{T})}=\left\|U^{n}\right\|_{l^{1} \rightarrow l^{1}} .
$$

The function $u$ is continuous and since $\left\|u^{n}\right\|_{L^{\infty}(\mathbb{T})} \leq\left\|u^{n}\right\|_{A(\mathbb{T})}$, it is clear that $|u(t)|=1$ for all $t \in \mathbb{T}$. (Otherwise the growth of the norms $\left\|U^{n}\right\|_{l^{1} \rightarrow l^{1}}$ would be exponential either as $n \rightarrow+\infty$ or as $n \rightarrow-\infty$.) Thus $u$ is a continuous function that maps $\mathbb{R}$ into the circle $\mathcal{C}=\{z \in \mathbb{C}:|z|=1\}$ on the complex plane $\mathbb{C}$. Every such function has the form $u(t)=e^{i \varphi(t)}$ where $\varphi: \mathbb{R} \rightarrow \mathbb{R}$ is a continuous function. 3 Since the function $u$ is $2 \pi$-periodic, we have $\varphi(t+2 \pi)=\varphi(t)(\bmod 2 \pi)$, i.e., $\varphi$ is a continuous mapping of the circle $\mathbb{T}$ into itself. We have $\left\|e^{i n \varphi}\right\|_{A(\mathbb{T})}=\left\|u^{n}\right\|_{A(\mathbb{T})}=\left\|U^{n}\right\|_{l^{1} \rightarrow l^{1}}$. Using our theorem, we obtain from $(20)$ that $\varphi(t)=\nu t+\varphi(0)$ for some $\nu \in \mathbb{Z}$. So $u(t)=\xi e^{i \nu t}$, where $\xi=e^{i \varphi(0)}$. Therefore, the operator $U$ is the operator of convolution with the sequence $\left\{u_{k}, k \in \mathbb{Z}\right\}$, where $u_{k}=0$ for all $k \neq \nu$ and $u_{\nu}=\xi$. Thus, $U=\xi S$, where $\xi \in \mathbb{C},|\xi|=1$, and $S$ is a translation $\left(S:\left\{x_{k}\right\} \rightarrow\left\{x_{k-\nu}\right\}\right)$.

2 . The theorem of the present work easily transfers to the multidimensional case. Let $A\left(\mathbb{T}^{d}\right)$ be the space of all continuous functions on the $d$-dimensional torus $\mathbb{T}^{d}$ with absolutely convergent Fourier series. We put $\|f\|_{A\left(\mathbb{T}^{d}\right)}=$ $\|\widehat{f}\|_{l^{1}\left(\mathbb{Z}^{d}\right)}$, where $\widehat{f}$ is the Fourier transform on $\mathbb{T}^{d}$. If $\varphi$ is a continuous mapping $\mathbb{T}^{d} \rightarrow \mathbb{T}$ such that

$$
\left\|e^{i n \varphi}\right\|_{A\left(\mathbb{T}^{d}\right)}=o\left(\left(\frac{\log \log |n|}{\log \log \log |n|}\right)^{1 / 12}\right), \quad n \in \mathbb{Z}, \quad|n| \rightarrow \infty,
$$

then $\varphi$ is linear, i.e., $\varphi(t)=(\nu, t)+\varphi(0)$, where $\nu \in \mathbb{Z}^{d}$. (Here $(\nu, t)$ stands for the inner product of vectors $\nu \in \mathbb{Z}^{d}$ and $t \in \mathbb{T}^{d}$.) The multidimensional case

\footnotetext{
${ }^{3}$ The function $\varphi$ is the lifting of $u$ with respect to the covering $e: \mathbb{R} \rightarrow \mathcal{C}$ where $e(x)=e^{i x}$. The existence of $\varphi$ is guaranteed by the monodromy theorem, see, e.g., [14, Lecture 4, corollary from Theorem 1].
} 
easily reduces to the one-dimensional case by induction over the dimension. It suffices only to note the following. Let $f \in A\left(\mathbb{T}^{d+1}\right)$. For a fixed $x \in \mathbb{T}^{d}$ consider the function $f_{x}$ on $\mathbb{T}$ defined by $f_{x}(y)=f(x, y)$. Then $\left\|f_{x}\right\|_{A(\mathbb{T})} \leq$ $\|f\|_{A\left(\mathbb{T}^{d+1}\right)}$.

The corresponding version for operators $U$ acting in $l^{1}\left(\mathbb{Z}^{d}\right)$ also holds.

3. As is noted by B. Green and S. V. Konyagin [10, $\S 1]$, it is plausible that the estimate in their theorem can be improved. Perhaps $(\log \log N / \log N)^{1 / 3}$ on the right side in $(2)$ can be replaced with $1 / \log N$ (further improvement is impossible). This improvement would allow to replace the right side in the condition of our theorem with $o\left((\log \log |n|)^{1 / 4}\right)$. Apparently it is easier to obtain an improvement of estimate (2) with the replacement of the exponent $1 / 3$ with $1 / 2$; in this connection see the work of T. Sanders [15]. This would allow to replace the exponent $1 / 12$ in our theorem with $1 / 8$. As to the possibility to eliminate $\log \log N$ in the Green-Konyagin theorem, see [10, $\S 6]$. This would allow to eliminate $\log \log \log |n|$ in our theorem.

4. Kahane's conjecture remains unproved even under the additional assumption of $C^{1}$-smoothness of $\varphi$. In this connection note the following result of the author [6] (see also [7]): if $\gamma(n) \geq 0$ is an arbitrary sequence, $\gamma(n) \rightarrow+\infty$, then there exists a $C^{1}$-smooth non-linear mapping $\varphi: \mathbb{T} \rightarrow \mathbb{T}$ such that $\left\|e^{i n \varphi}\right\|_{A(\mathbb{T})}=O(\gamma(|n|) \log |n|)$.

5 . It is not clear if there exists a continuous non-linear mapping $\varphi: \mathbb{T} \rightarrow$ $\mathbb{T}$ satisfying

$$
\left\|e^{i n_{k} \varphi}\right\|_{A(\mathbb{T})}=O(1),
$$

for some unbounded sequence of integers $\left\{n_{k}\right\}$. If $\varphi$ is absolutely continuous (i.e., if the function $\varphi: \mathbb{R} \rightarrow \mathbb{R}$ with condition (1) is absolutely continuous on each interval of length $2 \pi$ ) then this is impossible. We shortly explained this in [6]. Here we shall give a detailed and simple proof.

Note first that if $g$ is a real measurable function on $\mathbb{T}$ such that $\widehat{e^{i \lambda g}} \in$ $l^{1}(\mathbb{Z})$ for all $\lambda \in \mathbb{R}$ and

$$
\sup _{\lambda \in \mathbb{R}}\left\|\widehat{e^{i \lambda g}}\right\|_{l^{1}(\mathbb{Z})}<\infty
$$

then $g$ is constant almost everywhere. One can see this as follows. We have $\widehat{e^{i g}} \in l^{1}(\mathbb{Z})$, therefore, $e^{i g}$ coincides almost everywhere with a certain continuous function $\xi$. Then $|\xi|=1$ almost everywhere and therefore everywhere. It follows that $\xi=e^{i \psi}$, where $\psi: \mathbb{T} \rightarrow \mathbb{T}$ is a continuous mapping (this has already been explained in the first remark). Then for each $n \in \mathbb{Z}$ we have $e^{i n \psi}=\xi^{n}=e^{i n g}$ almost everywhere, therefore, $\left\|e^{i n \psi}\right\|_{A(\mathbb{T})}=\left\|\widehat{e^{i n g}}\right\|_{l^{1}(\mathbb{Z})}=$ 
$O(1), n \in \mathbb{Z}$. By the Beurling-Helson theorem the function $\psi$ is linear with integer slope. Thus, $g$ almost everywhere coincides mod $2 \pi$ with a linear function that has an integer slope. Let us take a (real) irrational number $\alpha$. Repeating the arguments for the function $g_{\alpha}=\alpha g$ instead of $g$, we see that $\alpha g$ is also almost everywhere coincides mod $2 \pi$ with a linear function that has an integer slope. This is possible only in the case when $g$ is constant almost everywhere.

Let $\varphi: \mathbb{T} \rightarrow \mathbb{T}$ be an absolutely continuous mapping. Suppose that we have (21). Subtracting an appropriate linear function we can assume that $\varphi$ is a real $2 \pi$-periodic function on $\mathbb{R}$. We can also assume that $n_{k} \rightarrow \infty$. For arbitrary $\lambda \in \mathbb{R}, \lambda \neq 0$, and $k=1,2, \ldots$ define a function $g_{\lambda, k}$ on $\mathbb{R}$ by

$$
g_{\lambda, k}(t)=\frac{\varphi\left(t+\lambda / n_{k}\right)-\varphi(t)}{\lambda / n_{k}}
$$

As $k \rightarrow \infty$, we have $g_{\lambda, k} \rightarrow \varphi^{\prime}$ almost everywhere. Note that

$$
e^{i \lambda g_{\lambda, k}(t)}=e^{i n_{k} \varphi\left(t+\lambda / n_{k}\right)} e^{-i n_{k} \varphi(t)},
$$

whence, assuming that $\left\|e^{i n_{k} \varphi}\right\|_{A(\mathbb{T})} \leq c, k=1,2, \ldots$, we obtain $\left\|e^{i \lambda g_{\lambda, k}}\right\|_{A(\mathbb{T})} \leq$ $c^{2}$. Tending $k$ to $\infty$, we see that $\left\|\widehat{e^{i \lambda \varphi^{\prime}}}\right\|_{l^{1}(\mathbb{Z})} \leq c^{2}$ for all $\lambda \in \mathbb{R}$ (the case when $\lambda=0$ is obvious). Thus, the derivative $\varphi^{\prime}$ of the function $\varphi$ is constant almost everywhere. From the condition of absolute continuity it follows that $\varphi$ is linear.

Initially, the theorem obtained in the present work was somewhat weaker. I am grateful to S. V. Konyagin who turned my attention to Theorem 1.3 of the work [10]. This allowed to improve the result.

I am also grateful to $\mathrm{Yu}$. N. Kuznetsova for the help with the proof of the lemma on the sections (Lemma 3).

\section{References}

1. A. Beurling, H. Helson, "Fourier-Stieltjes transforms with bounded powers", Math. Scand., 1 (1953), 120-126.

2. J.-P. Kahane, Série de Fourier absolument convergentes, Springer-Verlag, Berlin-Heidelberg-New York, 1970. 
3. J.-P. Kahane, "Quatre leçons sur les homéomorphismes du circle et les séries de Fourier", in: Topics in Modern Harmonic Analysis, Vol. II, Ist. Naz. Alta Mat. Francesco Severi, Roma, 1983, 955-990.

4. Z. L. Leibenson, "On the ring of functions with absolutely convergent Fourier series", Uspehi Matem. Nauk, 9:3(61) (1954), 157-162 (in Russian).

5. J.-P. Kahane, "Sur certaines classes de séries de Fourier absolument convergentes", J. de Mathématiques Pures et Appliquées, 35:3 (1956), 249-259.

6. V. V. Lebedev, "Diffeomorphisms of the circle and the Beurling-Helson theorem", Functional analysis and its applications, 36:1(2002), 25-29.

7. V. V. Lebedev, "Quantitative estimates in Beurling-Helson type theorems", Sbornik: Mathematics, 201:12 (2010), 1811-1836.

8. V. V. Lebedev, "Estimates in Beurling-Helson type theorems: Multidimensional case", Mathematical Notes, 90:3 (2011), 373-384.

9. J.-P. Kahane, "Transformées de Fourier des fonctions sommables", Proceedings of the Int. Congr. Math., 15-22 Aug., 1962, Stockholm, Sweden, Inst. Mittag-Leffler, Djursholm, Sweden, 1963, pp. 114-131.

10. B. Green, S. Konyagin, "On the Littlewood problem modulo a prime", Canad. J. Math., 61:1 (2009), 141-164.

11. E. M. Stein and R. Shakarchi, Fourier analysis: An introduction (Princeton Lectures in Analysis v. I), Princeton Univ. Press, Princeton and Oxford, 2003.

12. W. M. Schmidt, Diophantine Approximation, Lect. Notes in Math. 785, Springer-Verlag, Berlin-Heidelberg-New York, 1980.

13. R. Larsen, An introduction to the theory of multipliers, Springer-Verlag, Berlin-Heidelberg-New York, 1971.

14. M. M. Postnikov, Leçons de géométrie. Semester IV. Géométrie différentielle, Mir, Moscow, 2001. 
15. T. Sanders, "The Littlewood-Gowers problem", Journal d' Analyse Mathematique, 101:1 (2007), 123-162.

V. V. LEBEDEV

Dept. of Mathematical Analysis

Moscow State Institute of Electronics

and Mathematics (Technical University)

E-mail address: lebedevhome@gmail.com 